\title{
Criminologie
}

\section{L'évolution du réseau social des femmes victimes de violence conjugale}

\section{The evolution of the social network of women victims of domestic violence \\ La evolución de la red social de mujeres víctima de violencia conyugal}

\author{
Anne-Marie Nolet, Carlo Morselli et Marie-Marthe Cousineau
}

Volume 52, numéro 2, automne 2019

URI : https://id.erudit.org/iderudit/1065865ar

DOI : https://doi.org/10.7202/1065865ar

Aller au sommaire du numéro

Éditeur(s)

Les Presses de l’Université de Montréal

ISSN

0316-0041 (imprimé)

1492-1367 (numérique)

Découvrir la revue

Citer cet article

Nolet, A.-M., Morselli, C. \& Cousineau, M.-M. (2019). L'évolution du réseau social des femmes victimes de violence conjugale. Criminologie, 52(2), 300-320.

https://doi.org/10.7202/1065865ar
Résumé de l'article

Cet article vise à analyser l'évolution du réseau social des femmes victimes de violence conjugale en référence au concept d'autonomie relationnelle, défini comme une liberté de choisir parmi un ensemble diversifié d'options. Des entrevues individuelles ont été réalisées avec 30 femmes victimes de violence conjugale, permettant de dégager quatre étapes à travers lesquelles leur réseau change dans le temps : la relation violente, la rupture, le suivi en maison d'hébergement et le post-suivi. Les données sont analysées à partir des mesures de contrainte et de contrainte dyadique, qui permettent de saisir dans quelle mesure les femmes sont enfermées dans leur réseau social et/ou dans des relations avec certains acteurs en particulier. La contrainte des femmes diminue avec la rupture et le passage en maison d'hébergement, puis augmente pour la période post-suivi. Les relations contraignantes, principalement négatives et mitigées pendant la relation violente et la rupture, deviennent de plus en plus positives avec le passage en maison d'hébergement et le post-suivi. Les implications de ces résultats pour l'intervention et la recherche sont discutées.
Tous droits réservés @ Les Presses de l’Université de Montréal, 2019
Ce document est protégé par la loi sur le droit d'auteur. L'utilisation des services d'Érudit (y compris la reproduction) est assujettie à sa politique d'utilisation que vous pouvez consulter en ligne. 


\title{
L'évolution du réseau social des femmes victimes de violence conjugale
}

\author{
Anne-Marie Nolet ${ }^{1}$ \\ Doctorat en criminologie \\ Professionnelle de recherche \\ Centre international de criminologie comparée \\ anne-marie.nolet@umontreal.ca \\ Carlo Morselli \\ Professeur titulaire \\ École de criminologie de l'Université de Montréal \\ carlo.morselli@umontreal.ca

\section{Marie-Marthe Cousineau} \\ Professeure titulaire à l'École de criminologie de l'Université de Montréal \\ Vice-doyenne à la Faculté des arts et des sciences de l'Université de Montréal \\ Directrice universitaire CRSH-Partenariat-Trajetvi \\ mm.cousineau@umontreal.ca
}

RÉSUMÉ - Cet article vise à analyser l'évolution du réseau social des femmes victimes de violence conjugale en référence au concept d'autonomie relationnelle, défini comme une liberté de choisir parmi un ensemble diversifié d'options. Des entrevues individuelles ont été réalisées avec 30 femmes victimes de violence conjugale, permettant de dégager quatre étapes à travers lesquelles leur réseau change dans le temps: la relation violente, la rupture, le suivi en maison d'hébergement et le post-suivi. Les données sont analysées à partir des mesures de contrainte et de contrainte dyadique, qui permettent de saisir dans quelle mesure les femmes sont enfermées dans leur réseau social et/ou dans des relations avec certains acteurs en particulier. La contrainte des femmes diminue avec la rupture et le passage en maison d'hébergement, puis augmente pour la période post-suivi. Les relations contraignantes, principalement négatives et mitigées pendant la relation violente et la rupture, deviennent de plus en plus positives avec le passage en maison d'hébergement et le post-suivi. Les implications de ces résultats pour l'intervention et la recherche sont discutées.

1. Centre international de criminologie comparée, Université de Montréal, C. P. 6128, succursale Centre-ville, Montréal (Québec), Canada, H3C 3J7.

Criminologie, vol. 52, nº 2 (2019) 
MOTS CLÉs - Réseau social, réseau personnel, violence conjugale, autonomie relationnelle, maison d'hébergement.

\section{Introduction}

Pour certaines auteures (Goodman, Banyard, Woulfe, Ash et Mattern, 2016; Goodman et Smyth, 2011), la meilleure façon d'assurer la sécurité des femmes victimes de violence conjugale à long terme est d'améliorer le réseau social dans lequel elles retournent après l'intervention en milieu formel. Invitées à se prononcer sur le sujet, des intervenantes se disent effectivement préoccupées par le réseau social des femmes qu'elles veulent aider, mais admettent trouver difficile la mise en place de telles interventions (Goodman et al., 2016). Ce besoin exprimé par les intervenantes fait écho au manque de littérature scientifique portant sur le réseau social des femmes victimes de violence conjugale. Dans l'état actuel des connaissances, il est difficile pour les intervenantes de développer des modèles d'intervention centrés sur les réseaux et fondés sur des données empiriques.

Les études portant sur le soutien social permettent certes d'appuyer la pertinence de centrer l'intervention sur le réseau des femmes: les victimes de violence conjugale se perçoivent moins soutenues que leurs homologues non victimes (Levendosky et al., 2004; Thompson et al., 2000), mais celles qui se perçoivent soutenues s'en sortent mieux que les autres (Bybee et Sullivan, 2006; Coker, Smith, Thompson, McKeown et Bethea, 2004; Levendosky et al., 2004; Tan, Basta, Sullivan et Davidson, 1995; Thompson et al., 2000). Le soutien social demeure cependant un concept très général, voire flou, qui ne permet pas de saisir les mécanismes à travers lesquels le soutien se produit à même les réseaux sociaux (Carpentier et White, 2001). Il ne permet ainsi pas d'aiguiller davantage les intervenantes dans leur désir de développer de plus amples interventions centrées sur le réseau social des femmes.

D'un angle plus dynamique, les processus relationnels sont abordés par les chercheurs s'intéressant aux actions des membres du réseau social des femmes ainsi qu'à l'influence de ces actions sur les femmes elles-mêmes (voir entre autres Baker, 1997; Latta et Goodman, 2011; Rose, Campbell et Kub, 2000). Les résultats de ces études soulignent que les réactions perçues comme négatives par les femmes peuvent amener ces dernières à se retirer de leurs relations. Malgré leur capacité à mettre en lumière les interactions des femmes, ces études ont pour 
limite d'appréhender les relations de manière restreinte: il s'agit essentiellement de comprendre des dyades. Ce faisant, elles ne permettent pas de comprendre le réseau des femmes globalement.

L'utilisation de l'analyse de réseaux permet d'adopter ce regard global. À ce jour, les analyses de réseaux demeurent toutefois quasi absentes de la littérature scientifique en violence conjugale. Quelques auteurs ont tenté d'intégrer cette méthodologie à leurs travaux (Hoff, 1990; Katerndahl, Burge, Ferrer, Becho et Wood, 2013), mais leurs résultats ont une portée limitée pour l'intervention.

Comparant les relations de femmes victimes de violence conjugale entre les périodes «pendant» et «après» leur relation violente, Hoff (1990) arrive à des résultats peu concluants, la proportion de relations positives restant stable, diminuant et augmentant dans des proportions semblables. Il est cependant possible que cette absence de résultats soit due à la petite taille de son échantillon $(n=5)$, ou à son utilisation d'une échelle standardisée alors que l'analyse de réseau permet l'utilisation de mesures précises telles la taille ou la contrainte structurelle. De telles mesures sont utilisées par Katerndahl et al. (2013) qui, comparant le réseau de femmes victimes à celui de femmes non victimes, analysent les relations de soutien données et reçues par leurs participantes, ainsi que les relations de soutien données de part et d'autre dans chacune des dyades de leur réseau. Ils arrivent à la conclusion que le réseau des femmes victimes est significativement plus petit et moins dense que celui des non-victimes. Les femmes victimes auraient donc moins de relations et des relations moins connectées entre elles que les non-victimes. Bien qu'intéressants, les résultats de cette étude sont peu féconds pour l'intervention. D'abord, la constitution du réseau des femmes n'est pas analysée: les auteurs ne font aucune distinction en ce qui concerne la place occupée par le conjoint violent ou en ce qui concerne la place des professionnels. Ensuite, le type de violence conjugale vécue par leurs participantes n'est pas précisé, ce qui, selon Johnson $(1995,2006,2008)$, pourrait rendre leurs résultats impossibles à interpréter. Finalement, le regard adopté est statique, alors qu'il appert de plus en plus essentiel de considérer la problématique de violence conjugale comme une dynamique. Un regard processuel permettrait de préciser les effets du passage en maison d'hébergement sur le réseau des femmes, les auteures ne s'entendant pas à ce sujet, certaines soutenant qu'il permet de briser l'isolement (Corbeil et Marchand, 2006; Dagenais, 2015), d'autres qu'il isole davantage (Goodman et Smyth, 
2011 ; Goodman et al., 2016; Olsen, 2014). Une telle information serait utile aux intervenantes qui, en comprenant mieux les effets de leurs interventions sur le réseau des femmes, pourraient s'ajuster.

Le présent article a donc pour objectif d'analyser l'évolution du réseau personnel des femmes victimes de violence conjugale. L'atteinte de cet objectif requiert l'utilisation d'un cadre théorique permettant de poser un regard global sur l'ensemble des acteurs qui composent le réseau des femmes tout en étant cohérent avec la littérature en violence conjugale. Remplissant ces exigences, le cadre théorique développé s'appuie sur le concept d'autonomie relationnelle.

\section{Le concept d'autonomie relationnelle en contexte de violence conjugale}

La violence conjugale est ici conçue en tant que violence coercitive et de contrôle (Johnson, 1995, 2006, 2008), la forme de violence la plus fréquemment rencontrée dans les échantillons recrutés en maison d'hébergement (Johnson, 2006). Ce type de violence se caractérise par une dynamique au sein de laquelle les victimes sont graduellement amenées à vivre en fonction des désirs et de la vision de leur agresseur. La perte d'autonomie en découlant, mentionnée par Pence et Paymar (1993), est sans doute la conséquence de la violence conjugale la plus englobante. L'autonomie et la perte d'autonomie des femmes se développent toutefois dans l'ensemble de leur réseau social. Dans cette section, cette vision est présentée à partir des écrits de Burt $(1992,2005)$ en analyse de réseaux et d'Oshana (2006) en philosophie, permettant de proposer une définition bidimensionnelle de l'autonomie relationnelle et de lui associer des conditions d'émergence.

\section{La théorie des trous structuraux}

La théorie des trous structuraux (Burt, 1992, 2005) est un incontournable pour qui s'intéresse à l'autonomie et à la contrainte des acteurs selon une perspective sur les réseaux sociaux. Elle part de la prémisse que la structure des relations sociales informelles est constituée de groupes et de personnes faisant le pont entre ceux-ci. Selon cette théorie, les personnes faisant le pont entre les groupes sont appelées «courtières» et le vide qu'elles comblent, «trou structural». 
Les courtières jouent un rôle important en ce qui a trait à la diversification de l'information. Burt (1992) explique que l'information circule mieux à l'intérieur des groupes qu'entre les groupes, si bien que l'information et les opinions finissent par y converger. En conséquence, le fait de s'adresser à n'importe quel membre d'un groupe est susceptible de donner accès à une information similaire. En étant en contact avec plusieurs groupes, les courtières ont un regard plus complet sur l'information, les opinions, les pratiques, etc., et elles sont en mesure d'en profiter et d'en faire profiter les différents groupes qu'elles fréquentent.

La fluidité avec laquelle l'information circule à l'intérieur des groupes et l'homogénéisation qui en découle fait en sorte que les groupes deviennent d'importantes sources de contrôle à l'égard des comportements de leurs membres (Burt, 2005). À force de discussion, chacun devient au courant des réactions des autres en ce qui a trait aux actions posées. Chacun devient aussi au courant des actions des uns et des autres et peut y réagir, contribuant au contrôle des comportements jugés déviants et à la cristallisation des normes en vigueur.

Considérant la redondance de l'information fournie par les membres des groupes, de même que le contrôle pouvant émerger de ces derniers, Burt (1992) considère que les relations redondantes sont des sources de contrainte. Alors que les acteurs investissent du temps dans leurs relations avec elles, les relations contraignantes fournissent peu de diversité et imposent un contrôle des comportements.

La position des personnes à l'intérieur de la structure relationnelle - leur réseau personnel - les place ainsi dans un contexte de contrainte ou d'autonomie. Une personne contrainte par son réseau a accès à une information peu diversifiée. En n'ayant pas d'autres options vers lesquelles se tourner lorsqu'elle anticipe des réactions négatives à l'égard des actions qu'elle aimerait poser, elle est aussi sujette à devoir répondre aux demandes des membres de son réseau. Ce n'est donc pas tant le fait d'avoir un réseau exempt de toute contrainte et de tout contrôle qui importe, mais bien le fait d'avoir des avenues permettant d'y échapper.

La théorie des trous structuraux a été développée pour expliquer l'avantage compétitif de certains acteurs en milieu organisationnel. Le contexte d'émergence et les principales applications de la théorie sont donc loin de la violence conjugale. Certaines applications de la théorie ne sont toutefois pas si loin de la problématique qui nous intéresse. Par exemple, une étude de Burt (2004) souligne que les courtières sont les personnes les plus créatives. Le processus par lequel les courtières 
analysent et synthétisent différents points de vue pour en venir à émettre une idée nouvelle n'est pas étranger aux femmes victimes de violence conjugale. Pour mettre fin à la violence, elles doivent redéfinir leur situation et, pour ce faire, elles ont besoin de discuter avec des gens ayant des visions diversifiées de la violence conjugale (Lempert, 1996; Liang, Goodman, Tummala-Narra et Weintraub, 2005). Elles doivent aussi se redéfinir elles-mêmes: après avoir longtemps agi en fonction de ce que leur conjoint ou leur famille attendaient d'elles, elles doivent apprendre à identifier qui elles sont et ce qu'elles veulent (Goodman et al., 2016). Un contexte d'autonomie structurelle peut être particulièrement propice à une telle redéfinition. Pour pouvoir réinventer leur vie, les femmes ont aussi besoin d'accéder à des ressources nombreuses, pertinentes et diversifiées pouvant répondre à leurs besoins tout aussi nombreux et variés (Lyon, Lane et Menard, 2008; Moe, 2007; Netto, Moura, Araujo, Souza et Silva, 2017). Encore ici, selon la théorie des trous structuraux, le fait d'avoir accès à différents groupes est susceptible d'offrir davantage de diversité que le fait d'évoluer au sein d'un réseau contraignant.

Ce qui se passe à l'intérieur de la structure influence aussi nécessairement l'autonomie des personnes. Leurs contacts les contraignent-ils à agir d'une manière ou d'une autre? Le modèle d'autonomie d'Oshana (2006) servira de base pour cette réflexion.

\section{Le modèle d'autonomie d'Oshana}

Pour Oshana (2006), une personne autonome est capable de choisir et d'agir en subissant un minimum d'interférences telles la coercition, la manipulation ou l'incapacité physique. Cette auteure développe différentes conditions de l'autonomie et parmi celles-ci, deux peuvent être caractérisées de relationnelles. La première est celle des opportunités disponibles aux personnes, qui doivent être nombreuses, variées et pertinentes en fonction de leurs objectifs.

La deuxième concerne les caractéristiques des relations des personnes autonomes. Selon elle, les personnes autonomes:

1) peuvent prendre des décisions différentes de celles prises par les personnes qui les influencent, sans risque de rétribution;

2) évoluent dans des relations qui préservent leur sécurité; 
3) ne sont pas tenues responsables des besoins, des attentes et des échecs d'autrui;

4) ne font pas l'objet de manipulation et de désinformation;

5) ne sont pas financièrement dépendantes d'autrui.

Blâmer autrui pour ses choix, transmettre de fausses informations ou interdire l'accès à l'emploi sont donc quelques exemples d'actions qui feraient, selon le modèle d'Oshana (2006), directement et objectivement entrave à l'autonomie des personnes.

Il ne fait pas partie des objectifs d'Oshana (2006) de comprendre l'interprétation des personnes relativement aux actions des membres de leur réseau. Selon Burt (2005), l'historique des interactions entre personnes informerait pourtant celles-ci au sujet des interactions à venir et déterminerait la ligne d'action individuelle. Si la relation entre deux personnes a un précédent positif, les interactions à venir sont vues comme étant peu risquées. Si toutefois la relation a un précédent négatif, les interactions à venir sont vues comme comportant un risque.

\section{Les dimensions du concept d'autonomie relationnelle}

Les écrits d'Oshana (2006) et de Burt $(1992,2005)$ convergent à deux égards. Les deux parlent de l'importance de l'accès à des options diversifiées pour l'autonomie des personnes. Les deux parlent aussi des contraintes qui peuvent être imposées par autrui sur les comportements des personnes. Deux dimensions constitutives de l'autonomie peuvent ainsi être dégagées de leurs travaux, soit l'accès à des options diversifiées et la liberté de saisir les options disponibles, permettant de définir l'autonomie relationnelle comme une liberté de choisir parmi un ensemble diversifié d'options.

Les dimensions de l'autonomie ayant été posées, des conditions d'émergence peuvent y être associées. Les écrits de Burt (1992, 2005) permettent d'abord de proposer que les personnes les plus autonomes soient celles ayant une faible contrainte structurelle. Une personne très contrainte par son réseau est particulièrement désavantagée. Non seulement a-t-elle accès à peu d'options (Burt, 1992, 2005), mais elle est aussi vulnérable en regard de la perte de liberté qu'elle pourrait subir si ses proches se révélaient en désaccord avec ses choix. Une personne peu contrainte par son réseau est, quant à elle, particulièrement bien positionnée. En ayant accès à des sources d'information non redondantes, elle a accès à une vision plus complète, ce qui lui permet de 
connaître plus d'options. Sa position la protège par ailleurs du contrôle potentiel provenant de ses sources de contrainte: si elle perçoit ou anticipe une perte de liberté, elle peut se tourner vers d'autres options. Les écrits d'Oshana (2006) permettent ensuite de proposer que les personnes autonomes soient entourées de personnes qui agissent de sorte à respecter leur liberté d'action et leur accès à des options diversifiées. Les écrits de Burt (2005) permettent finalement de proposer que les personnes autonomes aient des attentes positives à l'égard des acteurs et actrices qui les entourent. Si leurs attentes à l'égard d'autrui sont positives, les personnes sont libres de saisir les options qu'elles convoitent. Si toutefois leurs attentes sont négatives, elles ne sont pas libres d'agir.

\section{Méthodologie}

La présente étude ${ }^{2}$ repose sur une analyse du réseau social de femmes victimes de violence conjugale. Les données utilisées dans cet article ont toutes été collectées par le biais d'entrevues individuelles ${ }^{3}$.

\section{L'échantillon}

Afin de procéder au recrutement des participantes, les maisons d'hébergement ont d'abord été contactées par l'entremise des organisations qui les représentent sur le plan provincial (la Fédération des maisons d'hébergement pour femmes et le Regroupement des maisons pour femmes victimes de violence conjugale). Les maisons souhaitant participer contactaient l'auteure principale et le recrutement des participantes était ensuite organisé en collaboration avec les intervenantes.

Ainsi, 30 femmes (15 résidentes, 11 ex-résidentes et 4 femmes en suivi externe) recrutées dans quatre maisons d'hébergement à travers le Québec ont accepté de participer à l'étude. L’âge moyen des participantes au moment de l'entrevue est de 36 ans et la majorité d'entre elles était mères $(n=26)$. Elles sont distribuées de manière équilibrée en ce qui concerne leur milieu de vie: 17 d'entre elles habitent en milieu rural et 13 habitent en milieu urbain. La plupart sont nées au Québec

2. Le certificat assurant le caractère éthique de la recherche a été délivré le 8 juin 2015 par le Comité d'éthique de la recherche en arts et en sciences de l'Université de Montréal.

3. La thèse doctorale de laquelle découle cet article inclut aussi de l'observation participante, de l'analyse de dossier et une entrevue de groupe (Nolet, 2018). 
$(n=20)$. La durée moyenne de leur relation avec leur conjoint violent est de 9,4 ans. Elles ont subi de la violence psychologique (100\%), sociale $(84 \%)$, économique (81\%), physique $(77 \%)$ et sexuelle $(40 \%)$.

\section{Les entrevues}

Un générateur de noms a été utilisé afin de reconstituer le réseau des participantes. Cet outil est constitué d'une ou de plusieurs questions élicitant une liste de noms de personnes faisant partie d'un réseau. Alors qu'un générateur de noms en deux questions se montre généralement suffisant pour identifier les membres significatifs de réseaux personnels (Marin et Hampton, 2007), le contexte de violence conjugale nécessite que des questions soient ajoutées, notamment sur le plan des relations négatives (Trotter et Allen, 2009), des changements survenus et de l'apport spécifique des conjoints violents et des milieux d'intervention. Les six questions constituant le générateur de noms utilisé sont les suivantes:

1. Au cours des 12 derniers mois, avec qui avez-vous parlé de sujets importants pour vous?

2. Présentement, avec qui discutez-vous de sujets importants pour vous?

3. En plus des personnes déjà mentionnées, y a-t-il des personnes avec qui vous avez passé beaucoup de temps au cours des 12 derniers mois? Si oui, qui sont ces personnes?

4. En plus des personnes déjà mentionnées, y a-t-il des personnes à qui vous croyez que vous pourriez demander de l'aide si vous en aviez besoin (ex.: voisin, collègue, etc.)?

5. Avec votre accord, j'ajouterais maintenant les résidentes de la maison d'hébergement dans la liste de personnes puisque pendant votre séjour, elles sont des sources d'interactions et de soutien potentiel. Me permettez-vous de les ajouter à la liste de personnes?

6. Avec votre accord, j'ajouterais aussi votre conjoint ou ex-conjoint. Je l'ajouterais, car il se peut qu'il ait une influence sur votre réseau. Me permettez-vous de l'ajouter à la liste de personnes?

Les relations entretenues avec chacune des personnes mentionnées ont été qualifiées à partir d'un outil dont le développement a été entrepris par Morselli et Tremblay (2004a, 2004b), puis ajusté dans le cadre d'une recherche portant sur les expériences délinquantes des jeunes à 
Montréal (Morselli, Gariépy et Gagnon, 2016). L'instrument permet de mesurer différentes dimensions relationnelles (discussion, respect, confiance, soutien, conflit) auxquelles des dimensions propres au contexte de violence conjugale, inspirées notamment de l'outil utilisé par Hoff (1990), ont été ajoutées (demande d'aide, confidences, valorisation, approbation). Pour chacune des dimensions relationnelles, les participantes étaient invitées à se prononcer au sujet de relations données (ex.: «je fais confiance à cette personne») et reçues (ex.: «cette personne me fait confiance»). Pour chaque affirmation, les participantes étaient invitées à formuler leur degré d'accord sur une échelle ordinale allant de 0 (tout à fait en désaccord) à 3 (tout à fait en accord).

Les participantes étaient ensuite invitées à rapporter à qui, parmi l'ensemble des acteurs et actrices de leur réseau, chacun et chacune parlent. Le choix du réseau de discussion pour compiler les relations entre les membres du réseau des participantes est motivé par la théorie des trous structuraux, dans laquelle Burt (2005) souligne que la position de courtier atteint son maximum d'effet à l'intérieur des réseaux de discussions informelles.

Des réseaux ont aussi été reconstitués à partir d'informations recueillies de manière semi-dirigée alors que les participantes étaient invitées à s'exprimer plus amplement sur les changements survenus dans leurs relations. La partie semi-dirigée des entrevues portait sur l'expérience des femmes en lien avec les membres de leur réseau (les actions de ceux-ci, la perception des femmes à cet égard, leurs propres actions, les changements survenus dans leur réseau).

\section{L'analyse}

Les données de réseaux ont été transposées dans des matrices relationnelles, permettant leur analyse à partir du logiciel UCINET 6. Le regard dynamique s'est imposé. Tentant d'abord de regrouper les réseaux en catégories telles que «résidentes» et «ex-résidentes», celles-ci sont apparues insuffisantes à représenter la diversité des étapes auxquelles étaient rendues les participantes. Par exemple, alors que certaines résidentes étaient en couple avec leur agresseur ou avaient rompu depuis quelques jours à peine au moment de l'entrevue, d'autres avaient rompu il y a plusieurs mois et avaient, depuis, tenté de se débrouiller par ellesmêmes. Quatre étapes ont finalement été dégagées des 30 entrevues, soit celle de la relation violente $(n=14)$, celle de la rupture avant le 
début du suivi des femmes en maison d'hébergement $(n=7)$, celle du suivi en maison d'hébergement $(n=15)$ et celle qui suit la fin du suivi en maison d'hébergement $(n=13)$. Puisque certaines femmes ont donné des informations sur plus d'une étape, 45 réseaux ont pu être reconstitués.

Le concept d'autonomie relationnelle a été présenté comme un concept bidimensionnel rattachant, d'un côté, la disponibilité d'options diversifiées et, d'un autre côté, la liberté de saisir les options disponibles. Trois conditions ont ensuite été associées à ces dimensions, à savoir la faible contrainte structurelle (Burt, 1992, 2005), les actions respectant l'autonomie des femmes (Oshana, 2006) et les attentes positives à l'égard d'autrui (Burt, 2005). Ces conditions peuvent être mesurées à partir d'indicateurs répartis en trois niveaux, bien que seulement deux niveaux soient analysés dans le cadre de cet article, soit les niveaux structurel et relationnel.

Sur le plan structurel, il s'agit de saisir la contrainte structurelle qui pèse sur les femmes. La principale mesure utilisée est celle de contrainte développée par Burt (1992), associée à la mesure de contrainte dyadique en découlant. Cette mesure de contrainte, permet de capter dans quelle mesure les participantes investissent dans des contacts qui sont euxmêmes en contact les uns avec les autres ou sont peu nombreux. Il s'agit donc d'indiquer dans quelle mesure les relations des femmes leur permettent d'avoir accès à des zones relationnelles diversifiées. La contrainte varie généralement entre 0 (faible contrainte) et 1 (forte contrainte), mais peut être de plus de 1 (De Nooy, Mrvar et Batagelj, 2005). La mesure de contrainte dyadique (Burt, 1992) constitue la force avec laquelle les membres d'un réseau le maintiennent enfermé: un acteur est contraint par un autre acteur dans la mesure où il n'a pas beaucoup de solutions de rechange à sa relation avec lui, et où les choix disponibles sont aussi en relation avec lui. Ce score varie, pour chaque dyade, entre 0 et 1 . La mesure de taille du réseau des femmes est utilisée en complémentarité puisqu'un réseau de taille très petite est nécessairement contraignant - étant rares, les options ne peuvent y être diversifiées.

Sur le plan relationnel, il s'agit, à partir de données qualitatives et de réseaux, de saisir la qualité des relations contraignantes des participantes. Les relations de confiance telles que rapportées par les femmes lors de la passation de l'interprétateur de noms (analyse de réseaux) sont considérées être des relations positives: les femmes ont des attentes positives à l'égard des actions des personnes en qui elles ont confiance 
(Burt, 2005; Rousseau, Sitkin, Burt et Camerer, 1998). Les personnes à l'égard desquelles les femmes ont des propos élogieux (analyse qualitative) sont aussi considérées comme des personnes à l'égard desquelles les femmes ont des attentes positives. Au contraire, lorsque les femmes ont rapporté ne pas faire confiance à leurs principales sources de contrainte, qu'elles ont rapporté un conflit avec elles ou qu'elles ne se sont prononcées que sur les méfaits causés par ces personnes, elles ont été catégorisées comme étant négatives. Finalement, lorsque les femmes ont rapporté des informations discordantes, les relations ont été considérées comme mitigées.

Afin d'analyser les tendances se dégageant du passage d'une étape à une autre, des tests de différence de moyenne non paramétriques (U de Mann Whitney) ont été utilisés.

\section{Limites}

Les analyses effectuées ne sont pas sans limites. Les étapes analysées ont été colligées en agrégeant des données plutôt qu'en suivant des tendances dans les trajectoires individuelles. Ce faisant, les résultats ne permettent pas de nuancer en ce qui a trait aux différentes trajectoires relationnelles possibles. La petite taille de l'échantillon, la très petite taille de certains sous-groupes et les écarts types parfois grands requièrent par ailleurs une attitude de prudence dans l'interprétation des résultats. Avant tout exploratoires, ces résultats ne peuvent être utilisés pour généraliser. Considérant la rareté des études telles que celles-ci, elle est néanmoins pertinente et utile, notamment pour aiguiller les chercheurs à venir qui souhaiteraient approfondir certains des résultats que voici.

\section{Le réseau social des femmes victimes de violence conjugale au temps de la relation violente, de la rupture, du suivi en maison d'hébergement et du post-suivi}

La Figure 1 présente la taille moyenne du réseau des participantes lors des quatre étapes dégagées des entrevues. Une augmentation de la taille peut être constatée à la suite de la rupture et pendant le suivi en maison d'hébergement, suivie d'une diminution pour la période post-suivi. Les tests de différence de moyenne non paramétriques montrent cependant 
des différences de moyenne non significatives entre les étapes « relation violente» et «rupture» (U M-W: 14; $\mathrm{p}<0,12$ ) de même qu'entre les étapes «rupture» et «suivi en maison d'hébergement» (U M-W : 29; $\mathrm{p}<0,21$ ), des résultats qui peuvent être dus à la très petite taille des sous-groupes aux deux premières étapes. La différence de moyenne s'avère cependant significative entre les étapes «relation violente» et «suivi en maison d'hébergement» (U M-W: 18,5; p < 0,003), de même qu'entre les étapes «suivi en maison d'hébergement» et "post-suivi» (U M-W : 27,5; $\mathrm{p}<0,001$ ).

FIGURE 1

Taille moyenne du réseau des participantes

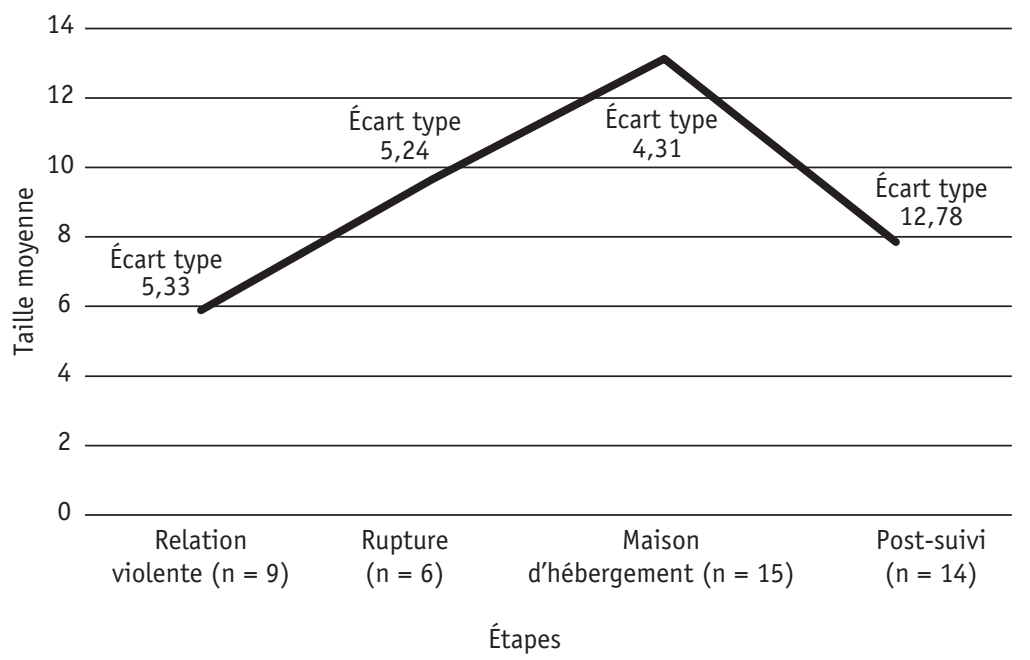

La contrainte qui pèse sur les participantes suit une tendance opposée à la taille (voir Figure 2). Elle diminue entre le moment de la relation violente et celui de la rupture, puis entre la rupture et le suivi en maison d'hébergement, ces diminutions s'avérant cependant non significatives d'un point de vue statistique (U M-W : 13,00; $p<0,1$ et U M-W : 34,00; $p<0,4$ respectivement). La différence de moyenne entre les étapes de la relation violente et du suivi en maison d'hébergement se révèle à l'inverse significative (U M-W: 19,00; $\mathrm{p}<0,004)$. En d'autres termes, tandis que la taille du réseau des participantes augmente, leur contrainte diminue. Cette relation entre les deux variables peut être expliquée par le fait que la mesure de contrainte est 
fortement influencée par la taille du réseau: plus un réseau est de grande taille, moins les personnes qui le composent sont susceptibles de se parler. Cette remarque mathématique est cependant insignifiante pour les femmes qui vivent concrètement la situation: l'élargissement de leur réseau vient, de fait, avec une plus faible redondance relationnelle, donc avec des relations susceptibles de leur apporter des options plus diversifiées en ce qui a trait aux ressources disponibles et aux différentes redéfinitions qui s'imposent. En outre, les femmes sont susceptibles de trouver en maison d'hébergement une vision de la violence conjugale différente de celle à laquelle elles ont accès avec leurs proches. Entre le suivi en maison d'hébergement et le post-suivi, la trajectoire s'inverse, la contrainte augmentant significativement ( $\mathrm{U} M-\mathrm{W}: 38,00 ; \mathrm{p}<0,003)$. C'est le retour à un réseau semblable, structurellement parlant, à celui de l'étape de la rupture.

F I G URE 2

Contrainte structurelle moyenne

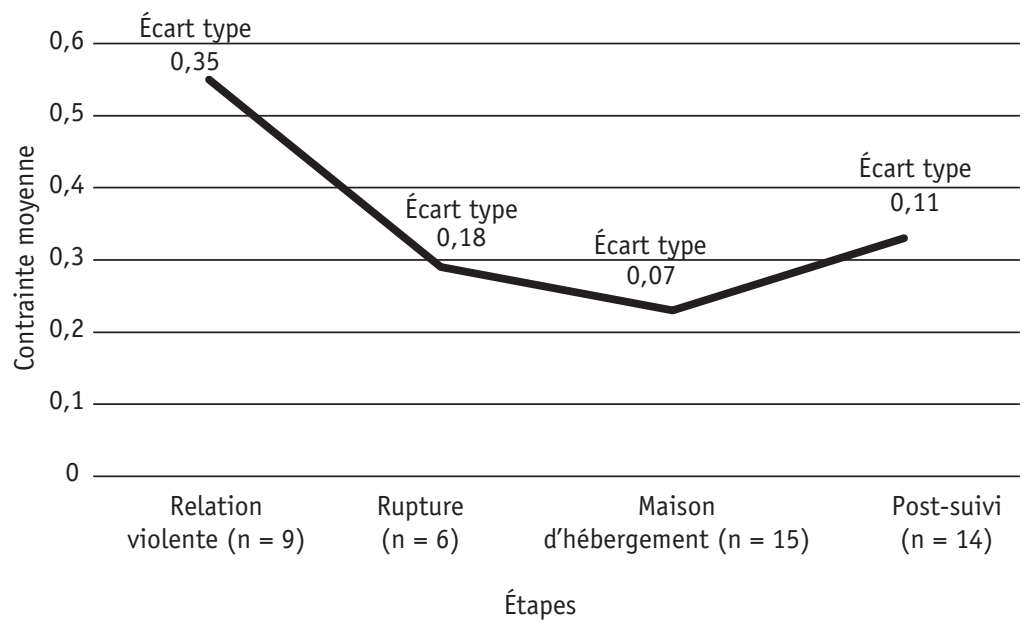

Il ne s'agit cependant pas d'un retour à la case départ. Alors que le conjoint violent est la principale source de contrainte de près de la moitié des participantes pendant la relation violente $(41,2 \%)$, il prend du recul après la rupture $(33,3 \%)$, jusqu'à devenir marginal pendant le suivi en maison d'hébergement $(4,8 \%)$ et à complètement disparaitre après le suivi. La diminution de la contrainte exercée par les conjoints 
violents se confirme par l'évolution de leur score moyen à la mesure de contrainte dyadique, qui passe de 0,25 (Écart type $=0,33$ ) à 0,02 (Écart type $=0,03$ ) entre les deux premières étapes, puis devient presque nul aux troisième et quatrième étapes, avec des scores moyens de 0,005 (Écart type $=0,009$ ) et 0,003 (Écart type $=0,08$ ) respectivement. Bien sûr, le fait que le conjoint violent ne constitue plus la source principale de contrainte n'implique pas qu'il soit complètement écarté de la vie des femmes ou qu'il ne fasse plus de tort: une violence post-séparation peut avoir lieu malgré la diminution de l'importance de l'agresseur dans le réseau des femmes. Néanmoins, le passage des femmes en maison d'hébergement fait en sorte qu'il ne domine plus leur réseau.

Pour bien comprendre les différences entre les étapes dégagées des entrevues avec les participantes, il importe par ailleurs de considérer l'évolution de la qualité de leurs relations contraignantes, c'est-à-dire des relations dans lesquelles elles investissent le plus. La Figure 3 présente la proportion de relations contraignantes positives, négatives et mitigées pour chacune des étapes étudiées. Lors des étapes de la relation violente et de la rupture, le réseau des femmes est dominé par des relations négatives et mitigées; en lien avec le cadre théorique développé, il est possible de croire qu'elles ont alors des attentes négatives à l'égard de ceux qui dominent leur réseau. Le suivi en maison d'hébergement marque la diminution de la proportion de relations contraignantes négatives et mitigées, qui sont alors remplacées par des relations positives. La fin du suivi en maison d'hébergement marque la fin des relations négatives et mitigées. Après leur passage en maison d'hébergement, les femmes peuvent conserver certaines relations négatives ou mitigées dans leur réseau, mais les relations dans lesquelles elles investissent le plus sont des relations à l'égard desquelles elles sont optimistes.

Le fait que le réseau des femmes est dominé par des relations positives pour la période post-suivi permet de conclure à une certaine pérennité des effets de l'intervention en maison d'hébergement: après le suivi, les femmes deviennent plus sélectives dans leurs relations. Rappelons toutefois que la contrainte augmente pendant cette période. Cette augmentation, même associée à des relations contraignantes positives, demeure préoccupante, puisqu'elle implique que les femmes ont accès à des relations et à des options aussi peu diversifiées qu'au temps de leur relation violente. Elles sont alors plus susceptibles d'être forcées à répondre aux demandes de leurs sources de contrainte (Burt, 
FIG URE 3

Qualité des relations avec les principales sources de contrainte

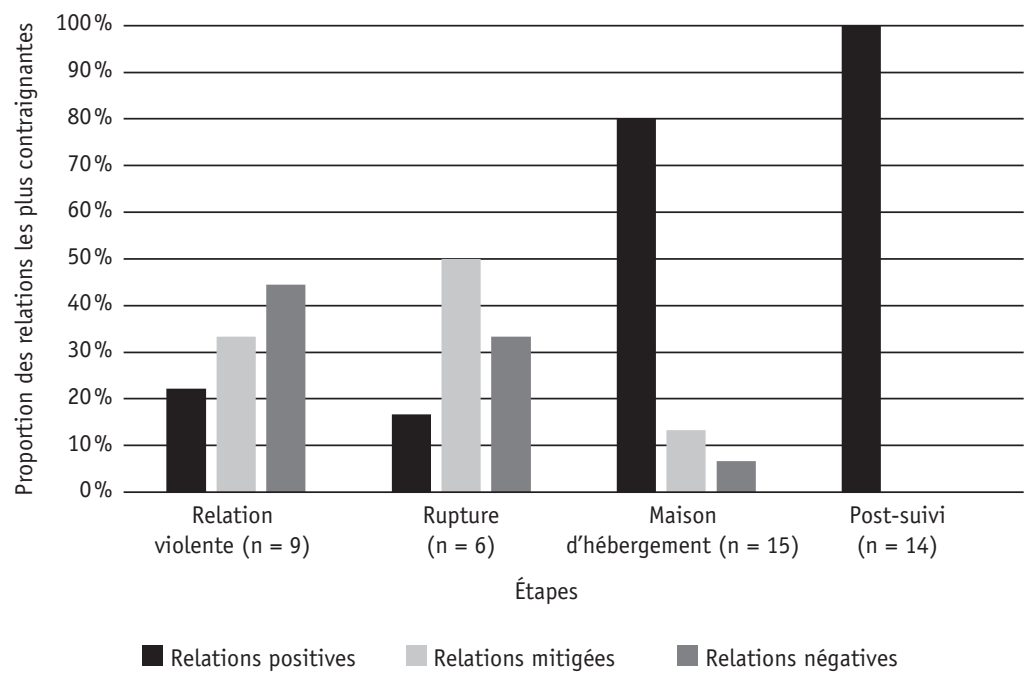

1992 ; 2005), leur laissant peu de marge de manœuvre dans des moments où elles voudraient agir d'une manière qui diffère des attentes de leurs proches à leur égard.

\section{Conclusion}

Dans le livre de Hoff (1990), le chapitre portant sur les changements survenus dans le réseau des femmes victimes de violence conjugale entre les moments «pendant» et «après» la relation violente présentait des conclusions discordantes. En procédant au découpage des étapes a posteriori et de manière inductive, la présente recherche a permis de déterminer quatre étapes significatives à partir desquelles se produisent les changements dans la structure et la qualité des relations des femmes. Cette avancée permet d'aiguiller les recherches à venir afin qu'elles puissent valider la pertinence des étapes dégagées et qu'elles testent les changements observés dans la présente étude. Plutôt que d'agréger les données des participantes, une analyse des trajectoires individuelles devrait alors être priorisée. Une approche par calendrier de vie semble réaliste et peu risquée pour les participantes. Il s'agirait de recruter les femmes au moyen des maisons d'hébergement et de faire le portrait de 
leur réseau, tel qu'elles s'en souviennent, pour les quatre étapes suggérées. Des items permettant d'évaluer le sentiment de soutien reçu à chacune des étapes pourraient être ajoutés de sorte à saisir les configurations permettant aux femmes de se sentir soutenues. Une hypothèse pourrait être posée à l'effet que les réseaux riches en autonomie seraient ceux dans lesquels les femmes se sentent le plus soutenues.

Les résultats présentés dans cet article ont l'avantage particulier de poser les effets actuels des maisons d'hébergement sur le réseau des femmes qui y sont suivies, cette connaissance étant nécessaire au plus ample développement des pratiques. À la lumière des analyses effectuées, le passage des femmes en maison d'hébergement est associé à une augmentation de leur autonomie relationnelle: pendant leur séjour, leur contrainte structurelle diminue fortement et les relations dans lesquelles elles investissent le plus sont principalement positives. Leurs relations contraignantes demeurent positives lors du post-suivi, laissant supposer une certaine pérennité des interventions et la préservation de leur capacité à saisir les options auxquelles elles ont accès. La contrainte structurelle des femmes augmente cependant lors de la période postsuivi, sous-entendant une diminution de leur accès à des options diversifiées. Cette augmentation de la contrainte structurelle pour la période post-suivi est normale puisqu'en quittant les maisons, les femmes perdent plusieurs sources d'interactions. Nous osons cependant proposer qu'il soit possible d'éviter ou, à tout le moins, de minimiser ce changement par la mise en place d'interventions centrées sur le réseau des femmes.

À cet égard, les premières phrases de cet article présentaient le constat de Goodman et al. (2016) en lien avec l'intervention centrée sur les réseaux en contexte de violence conjugale: les intervenantes souhaitent intégrer cette notion à leur pratique, mais rapportent vivre des difficultés lorsqu'elles tentent de le faire. Le cadre théorique développé, associé aux résultats présentés, permet de proposer l'utilisation des dimensions de l'autonomie relationnelle (accès à des options diversifiées et liberté de les saisir) en tant qu'objectifs à atteindre. Après avoir aidé les femmes à analyser leur réseau et à évaluer leurs besoins relationnels, les intervenantes pourraient améliorer l'accès des femmes à des options diversifiées en dirigeant les femmes vers certains services, en les aidant à reconnecter avec des proches ou en les appuyant dans le développement de nouvelles relations. Elles pourraient aussi augmenter la liberté des femmes de saisir les options qui leur sont accessibles, notamment 
en intervenant directement auprès de leurs proches, lorsque les femmes le souhaitent et le demandent, de sorte qu'ils apprennent à agir d'une manière qui suscite les attentes possibles des femmes. À cette fin, les proches peuvent être sensibilisés à l'égard de la violence conjugale, mais aussi à l'égard du rôle qu'ils jouent dans l'autonomisation des femmes.

Le développement d'une telle pratique centrée sur le réseau des femmes constitue une occasion, pour les maisons d'hébergement, de développer une nouvelle expertise, qui de plus est une expertise rare pouvant leur ouvrir des portes en tant que formatrices auprès d'autres maisons, voire d'autres organisations.

\section{Références}

Baker, P. L. (1997). And I went back: Battered women's negotiation of choice. Journal of Contemporary Ethnography, 26(1), 55-74.

Burt, R. S. (1992). Structural holes: The social structure of competition. Cambridge, MA: Harvard University Press.

Burt, R. S. (2004). Structural holes and good ideas. American Journal of Sociology, 110(2), 349-399.

Burt, R. S. (2005). Brokerage and closure: An introduction to social capital. Oxford, Royaume-Uni: Oxford University Press.

Bybee, D. et Sullivan, C. M. (2005). Predicting re-victimization of battered women 3 years after exiting a shelter program. American Journal of Community Psychology, 36(1/2), 85-96.

Carpentier, N. et White, D. (2001). Le soutien social: mise à jour et raffermissement d'un concept. Dans H. Dorvil et R. Mayer (dir.), Problèmes sociaux - Tome 1. Théories et méthodologies (p. 278-304). Sainte-Foy, Québec: Presses de l'Université du Québec,

Coker, A. L., Smith, P. H., Thompson, M. P., McKeown, R. E. et Bethea, L. (2004). Social support protects against the negative effects of partner violence on mental health. Journal of Women's Health $\mathcal{F}$ Gender-Based Medicine, 11(5), 465-476.

Corbeil, C. et Marchand, I. (2006). Penser l'intervention féministe à l'aune de l'approche intersectionnelle: défis et enjeux. Nouvelles pratiques sociales, 19(1), 40-57.

Dagenais, J. (2015). L'intervention féministe: les propos de celles qui l'ont pratiquée (Mémoire de maîtrise inédit). Université du Québec à Montréal.

De Nooy, W., Mrvar, A. et Batagelj, V. (2005). Exploratory social network analysis with Pajek. Cambridge, Royaume-Uni: Cambridge University Press.

Goodman, L. A., Banyard, V., Woulfe, J., Ash, S. et Mattern, G. (2016). Bringing a network-oriented approach to domestic violence services: A focus group exploration of promising practices. Violence Against Women, 22(1), 64-89. 
Goodman, L. A. et Smyth, K. F. (2011). A call for a social network-oriented approach to services for survivors of intimate partner violence. Psychology of Violence, 1(2), 79-92.

Hoff, L. A. (1990). Battered women as survivors. Londres, Royaume-Uni: Routledge.

Johnson, M. P. (1995). Patriarchal terrorism and common couple violence: Two forms of violence against women. Journal of Marriage and Family, 57(2), 283294.

Johnson, M. P. (2006). Conflict and control: Gender symmetry and asymmetry in domestic violence. Violence Against Women, 12(11), 1003-1018.

Johnson, M. P. (2008). Intimate terrorism, violent resistance and situational couple violence. Hanover, $\mathrm{NH}$ : Northeastern University Press.

Katerndahl, K., Burge, S., Ferrer, R., Becho, J. et Wood, R. (2013). Differences in social network structure and support among women in violent relationships. Journal of Interpersonal Violence, 28(9), 1948-1964.

Latta, R. E. et Goodman, L. A. (2011). Intervening in partner violence against women: A grounded theory exploration of informal network members' experiences. The Counselling Psychologist, 39(7), 973-1023.

Lempert, L. B. (1996). Women's strategies for survival: Developing agency in abusive relationships. Journal of Family Violence, 11(3), 262-289.

Levendosky, A. A., Bogat, G. A., Theran, S. A., Trotter, J. S., von Eye, A. et Davidson, W. S. (2004). The social networks of women experiencing domestic violence. American Journal of Community Psychology, 34(1/2), 95-109.

Liang, B., Goodman, L., Tummala-Narra, P. et Weintraub, S. (2005). A theoretical framework for understanding help-seeking processes among survivors of intimate partner violence. American Journal of Community Psychology, 36(1/2), 71-84.

Lyon, E., Lane, S. et Menard, A. (2008). Meeting survivors' needs: A multi-state study of domestic violence shelter experiences, final report. National Institute of Justice.

Marin, A. et Hampton, K. N. (2007). Simplifying the personal network name generator: Alternatives to traditional multiple and single name generators. Field Methods, 19(2), 163-193.

Moe, A. M. (2007). Silenced voices and structured survival: Battered women's help-seeking. Violence Against Women, 13, 676-699.

Morselli, C. et Tremblay, P. (2004a). Délinquance, performance et capital social: une théorie sociologique des carrières criminelles. Criminologie, 37(2), 89-122.

Morselli, C. et Tremblay, P. (2004b). Criminal achievement, offender networks and the benefits of low self-control. Criminology, 42(3), 773-804.

Morselli, C., Gariépy, A. et Gagnon, C. (2016). L'enchâssement social et la délinquance des pairs. Revue canadienne de criminologie et de justice pénale, 58 , 385-414.

Netto, L. A., Moura, M. A. V., Araujo, C. L. F., Souza, M. H. N. et Silva, G. F. (2017). Social support networks for women in situations of violence by an intimate partner, Texto Contexto Enferm, 26(2), 1-11. 
Nolet, A.-M. (2018). L'autonomie relationnelle des femmes victimes de violence conjugale: une analyse de leur réseau social (Thèse de doctorat inédite). Université de Montréal.

Olsen, L. (2014). Shelter rules: The good, the bad and the ugly. Washington State Coalition Against Domestic Violence.

Oshana, M. (2006). Personal autonomy in society. Burlington, VT: Ashgate.

Pence, E. et Paymar, M. (1993). Education groups for men who batter: The Duluth model. New York, NY: Springer.

Rose, L. E., Campbell, J. et Kub, J. (2000). The role of social support and family relationships in women's responses to battering. Health Care for Women International, 21(1), 27-39.

Rousseau, D. M., Sitkin, S. B., Burt, R. S. et Camerer, C. (1998). Not so different after all: A cross-discipline view of trust. The Academy of Management Review, 23(3), 393-404.

Tan, C., Basta, J., Sullivan, C. M. et Davidson, W. S. (1995). The role of social support in the lives of women exiting domestic violence shelter: An experimental study. Journal of Interpersonal Violence, 10(4), 437-451.

Thompson, M. P., Kaslow, N. J., Kingree, J. B., Rashid, A., Puett, R., Jacobs, D. et Matthews, A. (2000). Partner violence, social support, and distress among inner-city African American women. American Journal of Community Psychology, 28, 127-143.

Trotter, J. L. et Allen, N. E. (2009). The good, the bad, and the ugly: Domestic violence survivor experiences with their informal social networks. American Journal of Community Psychology, 43, 221-231.

\section{The evolution of the social network of women victims of domestic violence}

ABSTRACT - This article, based on the concept of relational autonomy defined as the freedom to choose between a diverse array of options, analyzes the evolution of a social network of women victims of domestic violence. Individual interviews were completed with 30 women and four stages in the development of social network were observed: violent relationship, break-up, shelter interventions, and post-shelter life. Network data was analyzed according to measures of constraints and dyadic constraints, which made it possible to identify the extent to which women are embedded in their social network and/or in relationships with specific actors. The constraints women face diminish at the time of break-up and as they enter a shelter and then increase after they exit the shelter. Close relationships, which were mainly negative or mixed during the stages of violent relationship and breakup, became increasingly positive during and after shelter life. The implications of these results for intervention and research are discussed.

KEYWORDS - Social networks, personal networks, domestic violence, intimate partner violence, relational autonomy, shelter. 


\section{La evolución de la red social de mujeres víctima de violencia conyugal}

RESUMEN - Este artículo analiza la evolución de la red social de mujeres víctima de violencia conyugal en referencia al concepto de autonomía relacional, definido como una libertad de escoger entre una diversidad de opciones. Se realizaron entrevistas individuales con 30 mujeres víctima de violencia conyugal, permitiendo identificar cuatro etapas a través de las cuales su red cambia con el tiempo: la relación violenta, la ruptura, el seguimiento en el refugio y el post-seguimiento. Los datos son analizados a partir de las medidas de restricción y de restricción diádica que permiten identificar en qué medida las mujeres están encerradas en su red social o en relaciones con ciertos actores en particular. La restricción de las mujeres disminuye con la ruptura y con el paso por el refugio, y luego aumenta en el periodo post-seguimiento. Las relaciones restrictivas, principalmente negativas y mitigadas durante la relación violenta y la ruptura, se vuelven cada vez más positivas con el paso por el refugio y durante el post-seguimiento. Las implicaciones de estos resultados para la intervención y la investigación serán discutidos.

PALABRAS CLAVE - Red social, red personal, violencia conyugal, autonomía relacional, refugio. 